\title{
Systematic evaluation of the gut microbiome of swamp eel (Monopterus albus) by 16S rRNA gene sequencing
}

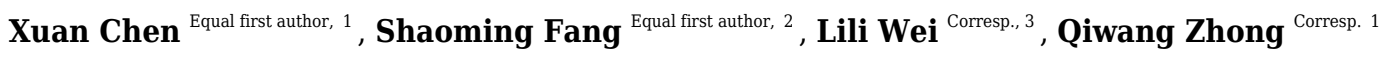 \\ 1 Jiangxi Engineering Laboratory for the Development and Utilization of Agricultural Microbial Resources, College of Biological Science and Engineering, \\ Jiangxi Agricultural University, Nanchang, China \\ College of Animal Science, Fujian Agriculture and Forestry University, Fuzhou, China \\ 3 College of Animal Science and Technology, Jiangxi Agricultural University, Nanchang, China \\ Corresponding Authors: Lili Wei, Qiwang Zhong \\ Email address: hbliliwei@163.com, zhongqw2000@163.com
}

\section{Background}

Swamp eel (Monopterus albus) is a commercially important farmed species in China. T he dysbiosis and homeostasis of gut microbiota might be associated with swamp eel's diseases pathogenesis and food digestion. The contribution of gut microbiome in fish growth and health is being increasingly recognized. However, little was known about the microbial community in the intestine of swamp eel (Monopterus albus).

\section{Methods}

The intestinal microbiomes of the five distinct gut sections (midgut content and mucosa, hindgut content and mucosa, and stools) of swamp eel were compared by the Illumina MiSeq sequencing of the bacterial 16S rRNA gene sequencing and statistical analysis.

\section{Results}

The results showed that the number of observed OTUs decreased from proximal to distal of intestine. PCoA analysis revealed significant separations among samples from different gut sections. Nevertheless, there were 54 core OTUs shared by all gut sections and 36 out of 54 core OTUs significantly varied in the abundances in different gut sections. Furthermore, we discovered 66 section-specific enriched KEGG pathways. These section-specific enriched microbial taxa (e.g., Bacillus, Lactobacillus) and potential function capacities (e.g., amino acid metabolism, carbohydrate metabolism) might play vital roles in nutrients metabolism, immune modulation, and host-microbe interactions of swamp eel.

\section{Conclusions}

Our results showed that the microbial diversity, composition, and function capacity were varied substantially in different gut sections. The gut section-specific enriched core microbial taxa and function capacities may exert an important role in swamp eel's nutrients metabolism, immune modulation, and host-microbe interactions. This study should provide basic insights into the gut microbiome of swamp eel. 
1 Systematic evaluation of the gut microbiome of swamp eel (Monopterus albus) by 16S rRNA

2 gene sequencing

3

4 Xuan Chen ${ }^{a \#}$, Shaoming Fang ${ }^{b \#}$, Lili Wei ${ }^{{ }^{*}}$, Qiwang Zhong ${ }^{a^{*}}$

6 a Jiangxi Engineering Laboratory for the Development and Utilization of Agricultural Microbial

7 Resources, College of Biological Science and Engineering, Jiangxi Agricultural University,

8 330045, Nanchang, China

$9{ }^{\mathrm{b}}$ College of Animal Science, Fujian Agriculture and Forestry University, 350002, Fuzhou, China

$10{ }^{\mathrm{c}}$ College of Animal Science and Technology, Jiangxi Agricultural University, 330045, Nanchang,

11 China

12

13 \# These authors contributed equally to the study

* Corresponding author 1: Qiwang Zhong

16 Tel\& Fax: +86-791-83813459

17 Postal address: College of Biological Science and Engineering, Jiangxi Agricultural University,

18 No. 1101 Zhimin Road, Nanchang 330045, P.R. China.

19 E-mail address: zhongqiwang@jxau.edu.cn

21 Corresponding author 2: Lili Wei

22 Tel\& Fax: +86-791-83853503

23 Postal address: College of Animal Science and Technology, Jiangxi Agricultural University, No.

241101 Zhimin Road, Nanchang 330045, P.R. China.

E-mail address: hbliliwei@163.com 


\section{Abstract}

\section{Background}

Swamp eel (Monopterus albus) is a commercially important farmed species in China. The dysbiosis and homeostasis of gut microbiota might be associated with swamp eel's diseases pathogenesis and food digestion. The contribution of gut microbiome in fish growth and health is being increasingly recognized. However, little was known about the microbial community in the intestine of swamp eel (Monopterus albus).

\section{Methods}

The intestinal microbiomes of the five distinct gut sections (midgut content and mucosa, hindgut content and mucosa, and stools) of swamp eel were compared by the Illumina MiSeq sequencing of the bacterial 16S rRNA gene sequencing and statistical analysis.

\section{Results}

The results showed that the number of observed OTUs decreased from proximal to distal of intestine. PCoA analysis revealed significant separations among samples from different gut sections. Nevertheless, there were 54 core OTUs shared by all gut sections and 36 out of 54 core OTUs significantly varied in the abundances in different gut sections. Furthermore, we discovered 66 section-specific enriched KEGG pathways. These section-specific enriched microbial taxa (e.g., Bacillus, Lactobacillus) and potential function capacities (e.g., amino acid metabolism, carbohydrate metabolism) might play vital roles in nutrients metabolism, immune modulation, and host-microbe interactions of swamp eel.

\section{Conclusions}

Our results showed that the microbial diversity, composition, and function capacity were varied substantially in different gut sections. The gut section-specific enriched core microbial taxa and function capacities may exert an important role in swamp eel's nutrients metabolism, immune modulation, and host-microbe interactions. This study should provide basic insights into the gut 
55

microbiome of swamp eel.

\section{Key words: Monopterus albus; $16 \mathrm{~S}$ rRNA sequencing; gut microbiome; function capacities}

\section{Introduction}

Swamp eel (Monopterus albus), taxonomically belonging to order Synbranchiformes, family Synbranchidae, is an air-breathing teleost and widely distributed in swamps, streams, ponds, and ricefields in areas of southern China, Japan, India and other Southeast Asian countries (FishBase, http://fishbase.org/). Due to its great growth performance and rich nutrient content, swamp eel has become a commercially important farmed species in China (Li et al., 2017), and in 2017 the production of swamp eel reached 358,295 tons. Diseases and low feed efficiency are two major factors restricting the development of swamp eel aquaculture. Probiotics are live microorganisms that when administered in adequate amounts confer a health benefit on the host (De et al., 2014). Many studies have revealed that the administration of probiotics could modulate gut microbial balance, enhance immune status, reduce disease susceptibility and improve feed efficiency (Caruffo et al., 2016; Hai, 2015; Newaj-Fyzul \& Austin, 2015). It suggested that the dysbiosis and homeostasis of gut microbiota might be associated with swamp eel's diseases pathogenesis and food digestion.

Earlier researches on gut microbiota of freshwater and marine fishes had demonstrated that gut microbiota played a crucial role in host nutrients metabolism, growth and health. Many cellulosedecomposing bacteria were harbored in the intestine of grass carp (Ctenopharyngodon idellus), such as Anoxybacillus, Actinomyces, and Citrobacter (Wu et al., 2012). Pompano (Trachinotus blochii) showed high abundance of Clostridia which was associated with polysaccharides degradation, when fed with commercial pellet (Rasheeda et al., 2017). The alpha diversity and the dominant bacterial taxa significantly changed with the development of Siniperca chuatsi (Yan et al., 2016). The interactions between threespine stickleback (Gasterosteus aculeatus) and gut microbiota played a key role in the development of gut innate immunity (Small et al., 2017). Moreover, gut microbial communities in the different gut sections exhibited distinct differences in diversity and richness. The significantly higher alpha-diversity indices in the midgut (named as 
82 foregut in Ye et al., 2014) than the hindgut in both Asian silver carp and gizzard shad. In salmon, microbial richness was higher in the digesta than in the mucosa (Gajardo et al., 2016), however, in the rabbitfish (Siganus fuscescens), the microbial richness significantly increased from content section to mucosal section (Nielsen et al., 2017). Since gut microbiome are complex and dynamic communities which have profound influences on fishes, it is important to systematically characterize the bacterial communities in different gut sections. However, to the best of our knowledge, there is few study about the gut microbiome of swamp eel.

Monopterus albus is a strict carnivore that prey on fishes, worms, crustaceans and other small aquatic animals in the wild (Liem, 1967). Under captive conditions, swamp eel are usually fed with surimi or mixed with commercial power feed. The gastrointestinal tract of swamp eel is a straight, uncoiled tube, passing directly to the anus, including the pharynx, esophagus, stomach, midgut (ileum) and hindgut (rectum). Bile enters the midgut by way of a short ductus choledochus (Liem, 1967). There is no external demarcation between mid- and hindgut, and histologically, the midgut and the hindgut are also similar that both of which contain four tunics: mucosa, submucosa, muscularis, and serosa. However, there are some microscopic di $\square$ erences. First, the mucosal folds of the midgut are in a reticular configuration, however, the mucosal folds of the hindgut are distinctly longitudinal and not as numerous as in the midgut. Second, in the midgut, the mucus secreting goblet cells are extremely numerous, while in the hindgut, the number of goblet cells is a food dependent feature, and starvation can cause a pronounced decrease in rectal goblet cells. Third, the serosa of the hindgut is much more prominent than that of the midgut. These make swamp eel likely to contain specific intestinal microbiota similar to those of carnivorous fish, such as the phylum Cetobacterium, Clostridium, Fusobacteria. Furthermore, the microscopic di $\square$ erences of gut may result in the different microbial structures in the midgut and hindgut.

The main objective of this study was to investigate gut microbial structures, compositions and function capacities of different gut sections of swamp eel using 16S rRNA gene sequencing. We wondered whether the different structural and functional characteristics of gut microbial community in different sections were correlated with swamp eel's nutrients metabolism, immune 
modulation, and host-microbe interactions. This study would provide the first glimpse of gut microbiome of swamp eel.

\section{Methods}

\section{Sample collection}

Swamp eels (40-45 g) were sampled from a commercial swamp eel farm in the Jiangxi Province, China $(28.4219 \mathrm{~N}, 116.4126 \mathrm{E})$, and acclimated in dechlorinated tap water at $25{ }^{\circ} \mathrm{C}$ in the $10 \mathrm{~L}$ aquarium tanks. Then the swamp eel individuals were fed with minced fish once a day for 8 weeks until dissection. The dechlorinated tap water was changed every day. All experimental swamp eels were healthy and had not received any antibiotics, probiotics or prebiotics during the feeding period. Fecal samples were collected immediately and separately before euthanasia. Fish were anesthetized with tricaine methanesulfonate and the whole intestines were aseptically removed from the abdominal cavity. The intestine was further dissected using sterile instruments to separate the midgut (immediately after the stomach) and the hindgut (immediately before the anus) sections according to Liem (Liem, 1967). The contents in each gut section were squeezed out and collected separately. The proximal and distal of the intestine were then washed with sterile PBS three times to remove remnants of the gut content. The gut mucosa was then scraped off with sterilized forcep and transferred into a microcentrifuge tube. All Samples from different gut sections were separately used for sequencing. All animal procedures were conducted according to the guidelines for the care and use of experimental animals established by the Ministry of Agriculture of China (No. SCXK YU2005-0001). Animal Care and Use Committee (ACUC) in Jiangxi Agricultural University specially approved this study.

\section{DNA extraction and 16S rRNA gene sequencing}

Total DNA was extracted from gut content and gut mucosa of different individuals using the PowerSoil ${ }^{\circledR}$ DNA Isolation Kit (Mo Bio, San Diego, CA, USA) according to the manufacturer's instruction. Fecal DNA extraction was performed using the QIAamp Stool Mini Kit (QIAGEN, Germany). The barcoded fusion forward primer 338F (5'-ACTCCTACGGGAGGCAGCA-3') and the reverse primer 806R (5'-GGACTACHVGGGTWTCTAAT-3') were used to amplify the 
136

137 138

139

140

141

142

143

144

145

146

147

148

149

150

151

152

153

154

155

156

157

158

159

160

161

162

V3-V4 hyper variable region of the $16 \mathrm{~S}$ rRNA gene. The Barcoded V3-V4 amplicons were sequenced using the paired-end method on Illumina MiSeq $2 \times 300$ platform (Illumina, USA) following the standard protocols.

\section{S rRNA gene sequencing data analysis}

To obtain the clean data, the barcodes and low quality sequences were filtrated using FASTXToolkit. FLASH software was used to merge high-quality paired-end reads into tags (Magoc \& Salzberg, 2011). Operational Taxonomic Unit (OTU) picking was performed using the USEARCH pipeline with a 97\% sequence identity (Edgar, 2010). We performed taxonomic assignments for the aligned sequences using the Ribosomal Database Project (RDP) classifier program with 80\% confidence threshold (Wang et al., 2007). Microbial taxa abundance and diversity indices were generated using Quantitative Insights Into Microbial Ecology (QIIME) (Caporaso et al., 2010). Phylogenetic investigation of communities by reconstruction of unobserved states (PICRUSt) was used to predict the functional profile of the microbial community (Langille et al., 2013). We extracted the closed reference OTU table from quality control reads in QIIME against the Greengenes database. OTU normalization, gene family abundances prediction, and function categorization based on KEGG (Kyoto Encyclopedia of Genes and Genomes) pathway was performed by PICRUSt according to the default settings.

\section{Statistical analysis}

Microbial species richness was analyzed using the observed number of OTUs. Principal Coordinate Analysis (PCoA) of the beta diversity was performed based on the unweighted and weighted distance matrix. Permutational Multivariate Analysis of Variance (PERMANOVA) was performed to identify section specific enriched microbial taxa and functional capacities (Nielsen et al., 2017). The output results were visualized using ggplot 2 and gplots in R package except the Venn diagrams which were drawn using the online tool (bioinformatics.psb.ugent.be/webtools/Venn/).

\section{Results}

Both data sets are accessible through NCBI's SRA, under study accession number SRP 
163

164

165

166

167

168

169

170

171

172

173

174

175

176

177

178

179

180

181

182

183

184

185

186

187

188

189

[145040].

\section{Microbial diversities and compositions in different gut sections}

At first, 405, 642, 227, 372 and 171 OTUs were identified in the midgut content, midgut mucosa, hindgut content, hindgut mucosa, and stools, respectively (Figure 1A). Then, we identified specific and common OTUs in different sections via a Venn diagram (Figure 1B-D). 63 common OTUs were detected among midgut content, hindgut content and stools. 315 OTUs were shared by both the midgut mucosa and hindgut mucosa. Importantly, we found 54 common OTUs as a core microbiota presented in all intestinal sections, while 53, 254, 5, 16, 37 specific OTUs were also detected for midgut content, midgut mucosa, hindgut content, hindgut mucosa, and stools, respectively. Moreover, PCoA analysis also revealed significant separations among samples from different gut sections (Figure 2, Figure S2).

To further uncover characteristics of microbial compositions in different gut sections, we analyzed the OTUs assigned for the phylum and genus level (Figure 3). At phylum level, Firmicutes, Fusobacteria, Proteobacteria, Bacteroidetes, and Actinobacteria were the five most dominant phyla. At genus level, Cetobacterium, Ralstonia, and Rhodococcus were the most predominant genera. Interestingly, the abundances of these microbial taxa changed significantly among different gut sections. For instances, Firmicutes occupied a large proportion of the gut microbiota in both midgut and hindgut regardless of the locations sample obtained, but it only occupied a small proportion of the gut microbiota in stools samples. Fusobacteria accounted for a higher proportion of gut microbiota in the content section than in the mucosal section. Cetobacterium was predominant in all samples, but a lower abundance in the midgut was observed when compared to the hindgut and stools. In contrast, the abundance of Rhodococcus in midgut was higher than that in hindgut and stools.

\section{Core microbial taxa enriched in different gut sections}

To identify which core microbial taxa showed different enrichment in specific gut section, we analyzed the abundance of the 54 core OTUs across all sections. As shown in Figure 4, total 36 section-specific enriched OTUs were observed. In the midgut content, seven enriched OTUs were 
annotated to Enhydrobacter, Comamonadaceae, Caulobacteraceae, Microbacteriaceae, Peptostreptococcaceae, Bradyrhizobium, and Deinococcus, respectively. Meanwhile, OTUs annotated to each of Roseburia, S24-7, Bacillus, Acidobacteria, Paracoccus, Lactococcus, and Oxalobacteraceae were enriched in the midgut mucosa. On the other hand, OTUs enriched in the hindgut content were annotated to Cetobacterium somerae, Arthrobacter, Coprococcus, Bacteroidaceae, Ruminococcaceae, Epulopiscium, and Citrobacter. OTUs annotated to Clostridium, Pseudomonas, Rhodococcus, Ralstonia, Achromobacter, Streptococcus, and Lactobacillus showed great abundance in the hindgut mucosa. Besides, 8 OTUs derived from Chryseobacterium, Comamonas, Serratia, Acinetobacter johnsonii, Pedobacter, Plesiomonas shigelloides, Pseudoxanthomonas Mexicana, and Aeromonadaceae increased in abundance in the stools samples.

\section{Comparison of microbial potential capacities in different gut sections}

To compare the potential functional capacity of microbial community in different gut sections, the relative abundances of KEGG pathways were predicted by PICRUSt. The results showed that 66 KEGG pathways exhibited significant differences in abundances across different gut sections (Figure 5). Among these, 26 pathways from the midgut samples, 28 pathways from the hindgut samples, and 12 pathways from stools samples. Notably, there were some characteristics of the distribution of differential pathways in specific gut section. For example, amino acid metabolism pathways such as lysine degradation, arginine and proline metabolism, and valine, leucine and isoleucine degradation were predominant in the midgut content. Cofactor and vitamins metabolism and signal transduction related pathways were overrepresented in the midgut mucosa. In the hindgut, carbohydrate and lipid metabolism pathways were prominent in the content, while bacterial replication, transcription, and translation related pathways were outstanding in the mucosa. In addition, we observed that microbial community was more capable of metabolizing secondary metabolites and xenobiotics in the stools.

\section{Discussion}

In this study, 16S rRNA sequencing analysis revealed the diversity, composition, and potential 
217 functional capacity of microbial community across different gut sections in swamp eel. To our best

218 knowledge, this is the first study systematically evaluating the gut microbiome of swamp eel

219 (Monopterus albus).

220 At phylum level, Firmicutes, Fusobacteria, Proteobacteria, Bacteroidetes, and Actinobacteria

221

222

223

224

225

226

227

228

229

230

231

232

233

234

235

236

237

238

239

240

241

242

243

were the five most dominant phyla. At genus level, Cetobacterium, Ralstonia, and Rhodococcus were the most predominant genera in the intestinal microbiota communities of swamp eel. Data analysis also showed that the most of the microbiome found in the intestine of swamp eel have been detected in other fish. This was in consistent with the results in Japanese eels (Huang et al., 2018). However, compared with Anguillid eel species, the intestinal microbial composition of swamp eel was markedly different. At the phylum level, Proteobacteria, Fusobacteria and Bacteroidetes were the dominant bacterial groups in European eels, and Proteobacteria is the most abundant phylum, accounting for $70.35 \pm 17.2 \%$ of the total number of reads (Huang et al., 2018). While in swamp eel, Proteobacteria accounted for only $12.88 \%$ of the total number of reads and were lower in hindgut mucosa and hindgut content, which accounted for $2.82 \%$ and $1.17 \%$ of the sequenced reads, respectively. The difference in genus is more obvious. Relative abundance of the top 5 genera in the intestinal mucosa of swamp eel were Cetobacterium, Ralstonia, and Rhodococcus, Mycobacterium, Clostridium. However, the top 5 bacterial genera in European eel intestine were Aeromonas, Cetobacterium, Plesiomonas, Shewanella, Paludibacter. Moreover, for the Giant-Mottled eels (Anguilla. marmorata) the dominant bacterial genera of were Acinetobacter, Mycoplasma and Shewanella. This difference may be related to the genetic characteristics of the species (Goodrich et al., 2014; Li et al., 2018). And also, the diet is one of the important factor that influences the community composition (Moschen et al., 2012; Piazzon et al., 2017).In this study, minced fish were used as feed, while the cultivated European eel were fed with commercial power feed and the Giant-Mottled eels were caught from the wild.

In this study, the number of observed OTUs decreased from the proximal to the distal section of intestine in swamp eel. This result was different from many vertebrate microbiome studies which revealed that the distal section of intestine had higher richness and diversity than the 
244

245

246

247

248

249

250

251

252

253

254

255

256

257

258

259

260

261

262

263

264

265

266

267

268

269

270

proximal section. This difference may be associated with the specific physiological structure of swamp eel's intestine. Unlike omnivorous and herbivorous fish which usually have long and coiled intestines (Pereira et al., 2015; Santos et al., 2016), swamp eel is carnivorous which intestine is short and straight. And, generally, the mid-gut is thought to be the organ where the majority of digestion occurs (Egerton et al., 2015). Considering the crucial role of gut microbiota in host nutrients metabolism, we speculated that much more microbes inhabitation in the proximal section of swamp eel's intestine should benefit to host fast digestion and absorption of nutrients. Furthermore, much more OTUs presented in the mucosal section than the content section regardless of the locations that samples obtained. This result was consistent with gut microbiome studies in rabbitfish (Siganus fuscescens) and loach (Nielsen et al., 2017; Yang et al., 2017) and reinforced previous findings that the mucosal section might serve as a reservoir of diverse bacterial species (Lu et al., 2014). OTUs assigned for the phylum level and genus level further revealed some specific features of microbial compositions of swamp eel. Previous study demonstrated that the high abundance of Firmicutes was observed in the gut microbiome of omnivorous fishes and Fusobacteria was the predominant phylum in the gut microbiome of carnivorous fishes (Liu et al., 2016). Here, the microbial communities of swamp eel were dominated by, in order, Firmicutes, Fusobacteria, Proteobacteria, Bacteroidetes, and Actinobacteria. Furthermore, we found that Firmicutes was more predominant in the midgut and hindgut than the stools, while the abundance of Fusobacteria was higher in the content section than the mucosal section. The most dominant genus Cetobacterium, Ralstonia and Rhodococcus also varied in the abundances across different gut sections. These results suggested that using samples from single gut section to represent an overview of gut microbiota would likely fail to detect community variation responding to physiological variations of the gut (Durbán et al., 2011).

Although the gut microbiota showed distinct spatial heterogeneity, we still identified a core microbiota consisting of 54 common OTUs in all gut section. It was in line with previous fishes gut microbiome studies indicating that specific microbial taxa could form a stable core microbota in the intestine (Baldo et al., 2015; Rudi et al., 2018). Furthermore, we found that the enrichments 
271 of these microbial taxa were associated with nutrients metabolism, immune modulation, and 272 habitat adaptions. In the content section, most of the enriched microbial taxa were associated with 273 nutrients metabolism. For example, dietary fiber degradation associated bacteria Enhydrobacter 274 and Comamonadaceae (Premalatha et al., 2015; Sakurai et al., 2017), and amino acid metabolism associated bacteria Caulobacteraceae and Microbacteriaceae (Yin et al., 2017) were enriched in the midgut content. Gut microbial taxa equipped with multiple carbohydrate active enzymes such as Bacteroidaceae, Ruminococcaceae, Coprococcus, and Citrobacter (Luo et al., 2017; Tap et al., 2015; Wu et al., 2012) which involved in non-digestible dietary carbohydrates metabolism showed great abundance in the hindgut content. Notably, Cetobacterium somerae as a vitamin B-12 and antimicrobial metabolites producing species had a higher abundance in the hindgut content. It was similar to the results of many other fish gut microbiota studies (Bledsoe et al., 2016; Larsen et al., 282 2014).

Interestingly, stools samples as an end product of nutrients metabolism in the content section, several potential aquatic pathogenic bacteria were enriched, including Serratia, Acinetobacter johnsonii, Plesiomonas shigelloides, and Aeromonadaceae (González et al., 2000; Martins et al., 2013; Nadirah et al., 2012; Huang et al., 2018). P. shigelloides is a cause of diarrhea in human, usually isolated from the faeces (Khan et al., 2004) and has also been found from the gut of many fishes, such as tilapia (Oreochromis niloticus) (Nadirah et al., 2012), largemouth bass Micropterus salmoides (Larsen et al., 2014) and Japanese eel Anguilla japonica (Hsu et al., 2018). In grass carps, $P$. shigelloides has also been found to be associated with muscle erosive disease (Hu et al., 2014). Acinetobacter johnsonii rescently were regarded as opportunistic pathogens for farmed rainbow trout (Kozińska et al., 2014) and blunt snout bream Megalobrama amblycephala (Cao et al., 2017). However, they did not cause any infections or diseases in our feeding swamp eels. It indicated that these bacteria were native inhabitants of swamp eel's stools, and the intestine maybe has a certain ability to enrich harmful bacteria into feces and excrete them out of the body.

Meanwhile, many immune modulation associated bacteria were found inhabiting in the mucosal section. For instance, $S 24-7$ modulated mucosal immune homeostasis and Roseburia regulated 
298

299

300

301

302

innate immunity (Liu et al., 2017; Patterson et al., 2017). Potential probiotics including Bacillus, Acidobacteria, and Lactococcus (Bernardeau et al., 2017; Lv et al., 2016; Wu et al., 2018) were predominant in the midgut mucosa and Lactobacillus were predominant in the hindgut mucosa. Clostridium and Lactobacillus involved in immune response, Pseudomonas and Achromobacter had strong antimicrobial activities, and Rhodococcus showed properties of probiotic (Nayak, 2010; Sharifuzzaman et al., 2017; Zothanpuia et al., 2016) were overrepresented in the hindgut mucosa. Besides, it was noteworthy that aerobic bacteria Bradyrhizobium, Deinococcus, Arthrobacter, and Comamonas preferred to thrive in the content section and anaerobes and obligate anaerobes such as Paracoccus, Ralstonia and Streptococcus were more prevalent in the mucosal section. This section-specific distributions might be related to its special respiration. Monopterus albus is an airbreathing teleost using the buccopharyngeal cavity for gas exchange (Damsgaard et al., 2014) and this is likely to cause a small amount of air into the intestine. Although there was no direct study on the oxygen concentration in the intestinal tract of Monopterus albus, early literature suggested that the intestine of Monopterus albus might have respiratory function (Petukat, 1965).

The potential functional capacities of microbial communities were distinctly different across different gut sections and these differential microbial functional capacities should be related to host physiological functions and host-microbes interactions. Amino acid metabolism pathways were more abundant in the midgut content, suggesting that gut microbiota in the midgut content may help swamp eel to digest dietary amino acids. Cofactors and vitamins metabolism and cellular signals processing pathways were enriched in the midgut mucosa. Since fishes lack the biosynthetic capacity for most vitamins, it is important that vitamins produced by gut microbiota will play a key role in host growth, intestinal mucosal immune and signaling molecules expression (Feng et al., 2016; Li et al., 2015). In the hindgut content, a high level of carbohydrate and lipid metabolism was identified. It was in line with previous studies that gut microbiome of fish hindgut had the ability of fermentation of non-digestible polysaccharides to short-chain fatty acids (SCFAs) (Geraylou et al., 2013; Mountfort et al., 2002). In the hindgut mucosa, microbial replications, transcriptions and translations related pathways were concentrated, which was in 
325

326

327

328

329

330

331

332

333

334

335

336

337

338

339

340

341

342

343

344

345 346

347

348

40

consistent with previous studies that hindgut mucosa was an essential gut region where interactions between gut microbiota and host cells happened (Morgan et al., 2015; Sellers \& Morton, 2014).

Intriguingly, we observed that microbial xenobiotics and secondary metabolites metabolism pathways were more predominant in the stools samples. This result and the enriched microbial taxa in stools above indicated that stools may service as a "wastes dump" of swamp eel and microbial community.

\section{Conclusions}

In the present study, we comprehensively characterized the microbial communities in different gut sections of swamp eel. Our results showed that the microbial diversity, composition, and function capacity were varied substantially in longitudinal and radial parts of the intestine. The microbial diversity and composition across different gut sections could reflect the characteristics of swamp eel's intestine structures and feeding habit. The gut section-specific enriched core microbial taxa and function capacities may exert an important role in swamp eel's nutrients metabolism, immune modulation, and host-microbe interactions. Taken together, these results should provide a basis for further research on gut microbiome of swamp eel.

\section{Conflict of interest}

There is no conflict of interest related to this research.

\section{Transparency document}

The Transparency document associated with this article can be found in the attachment.

\section{Acknowledgements}

This work was supported by grants from the National Natural Science Foundation of China (No. 
31160530 and No. 31360634), the Science and Technology Support Program of Jiangxi Province

(20122BBF60074) and the Project of Education Department in Jiangxi Province (GJJ13289).

\section{References:}

Baldo L, Riera JL, Tooming-Klunderud A, Albà MM, Salzburger W. 2015. Gut Microbiota Dynamics during Dietary Shift in Eastern African Cichlid Fishes. PLoS One 10(5):e0127462 DOI: 10.1371/journal.pone.0127462.

Bernardeau M, Lehtinen MJ, Forssten SD, Nurminen P. 2017. Importance of the gastrointestinal life cycle of Bacillus for probiotic functionality. Journal of food science and technology 54(8):2570-2584 DOI: 10.1007/s13197-017-2688-3.

Bledsoe JW, Peterson BC, Swanson KS, Small BC. 2016. Ontogenetic characterization of the intestinal microbiota of channel catfish through 16S rRNA gene sequencing reveals insights on temporal shifts and the influence of environmental microbes. PLoS One 11(11):e0166379 DOI:10.1371/journal.pone.0166379.

Cao H, Yu L, Ou R, Lu L, Yang X, Yang Y. 2017. Acinetobacter johnsonii: an emerging pathogen for cultured blunt snout bream Megalobrama amblycephala. Isr J Aquacult-Bamid 69(1368):1-7.

Caporaso JG, Kuczynski J, Stombaugh J, Bittinger K, Bushman FD, Costello EK, Fierer N, Peña AG, Goodrich JK, Gordon JI, Huttley GA, Kelley ST, Knights D, Koenig JE, Ley RE, Lozupone CA, McDonald D, Muegge BD, Pirrung M, Reeder J, Sevinsky JR, Turnbaugh PJ, Walters WA, Widmann J, Yatsunenko T, Zaneveld J, Knight R. 2010. QIIME allows analysis of high-throughput community sequencing data. Nature methods 7:335-336 DOI: 10.1038/nmeth.f.303.

Caruffo M, Navarrete NC, Salgado OA, Faúndez NB, Gajardo MC, Feijóo CG, Reyes-Jara A, García K, Navarrete P. 2016. Protective Yeasts Control V. anguillarum Pathogenicity and Modulate the Innate Immune Response of Challenged Zebrafish (Danio rerio) Larvae. Front Cell Infect Microbiol 6:127 DOI: 10.3389/fcimb.2016.00127

De BC, Meena DK, Behera BK, Das P, Das Mohapatra PK, Sharma AP. 2014. Probiotics in fish and shellfish culture: immunomodulatory and ecophysiological responses. Fish Physiol Biochem 40 (3):921-71 DOI 10.1007/s10695-013-9897-0.

Damsgaard C, Findorf I, Helbo S, Kocagoz Y, Buchanan R, Huong do TT, Weber RE, Fago A, Bayley M, Wang T. 2014. High blood oxygen affinity in the air-breathing swamp eel Monopterus albus. Comparative Biochemistry and Physiology Part A 178:102-8 DOI: 10.1016/j.cbpa.2014.08.001.

Durbán A, Abellán JJ, Jiménez-Hernández N, Ponce M, Ponce J, Sala T, D'Auria G, Latorre A, Moya A. 2011. Assessing gut microbial diversity from feces and rectal mucosa. Microbial ecology 61:123-133 DOI: 10.1007/s00248-010-9738-y.

Edgar RC. 2010. Search and clustering orders of magnitude faster than BLAST. Bioinformatics 26: 2460-2461 DOI: 10.1093/bioinformatics/btq461.

Egerton S, Culloty S, Whooley J, Stanton C, Ross RP. 2018. The Gut Microbiota of Marine Fish. Frontiers in Microbiology 9:873 DOI: 10.3389/fmicb.2018.00873. 
386

387

388

Feng L, Li SQ, Jiang WD, Liu Y, Jiang J, Wu P, Zhao J, Kuang SY, Tang L, Tang WN, Zhang YA, Zhou XQ. 2016. Deficiency of dietary niacin impaired intestinal mucosal immune function via regulating intestinal NFkappaB, Nrf2 and MLCK signaling pathways in young grass carp (Ctenopharyngodon idella). Fish \& shellfish immunology 49:177-93 DOI: 10.1016/j.fsi.2015.12.015.

Gajardo K, Rodiles A, Kortner TM, Krogdahl A, Bakke AM, Merrifield DL, Sorum H. 2016. A high-resolution map of the gut microbiota in Atlantic salmon (Salmo salar): A basis for comparative gut microbial research. Scientific reports 6: 30893 DOI: 10.1038/srep30893.

Geraylou Z, Souffreau C, Rurangwa E, Maes GE, Spanier KI, Courtin CM, Delcour JA, Buyse J, Ollevier F. 2013. Prebiotic effects of arabinoxylan oligosaccharides on juvenile Siberian sturgeon (Acipenser baerii) with emphasis on the modulation of the gut microbiota using 454 pyrosequencing. FEMS microbiology ecology 86(2):357-71 DOI: 10.1111/1574-6941.12169.

González CJ, Santos JA, García-López ML, Otero A. 2000. Psychrobacters and related bacteria in freshwater fish. Journal of food protection 63(3):315-21.

Goodrich JK, Waters JL, Poole AC, Sutter JL, Koren O, Blekhman R, Beaumont M, Van Treuren W, Knight R, Bell JT, Spector TD, Clark AG, Ley RE. 2014. Human genetics shape the gut microbiome. Cell 159(4):789799 DOI: 10.1016/j.cell.2014.09.053.

Hai NV. 2015. The use of probiotics in aquaculture. Journal of applied microbiology 119: 917-935 DOI: 10.1111/jam.12886.

Hsu H Yi, Chang FC, Wang YB, Chen SH, Lin YP, Lin CY, Han YS. 2018. Revealing the compositions of the intestinal microbiota of three Anguillid eel species using 16S rDNA sequencing. Aquaculture Research 49(7): 2404-2415.

Hu Q, Lin Q, Shi C, Fu X, Li N, Liu L, Wu S. 2014. Isolation and identification of a pathogenic Plesiomonas shigelloides from diseased grass carp. Wei Sheng Wu Xue Bao 54:229-235. (In Chinese).

Huang W, Cheng Z, Lei S, Liu L, Lv X, Chen L, Wu M, Wang C, Tian B, Song Y. 2018. Community composition, diversity, and metabolism of intestinal microbiota in cultivated European eel (Anguilla anguilla). Appl Microbiol Biotechnol 102(9):4143-4157 DOI: 10.1007/s00253-018-8885-9.

Khan AM, Faruque AS, Hossain MS, Sattar S, Fuchs GJ, Salam MA. 2004. Plesiomonas shigelloides-associated diarrhoea in Bangladeshi children: a hospital-based surveillance study. Journal of Tropical Pediatrics 50(6):354-356 DOI: 10.1093/tropej/50.6.354.

Kozińska A, Paździor E, Pękala A, Niemczuk W. 2014. Acinetobacter johnsonii and Acinetobacter lwoffii - the emerging fish pathogens. Bull Vet Inst Pulawy 58(2):193-199 DOI: 10.2478/bvip-2014-0029.

Langille MG, Zaneveld J, Caporaso JG, McDonald D, Knights D, Reyes JA, Clemente JC, Burkepile DE, Vega Thurber RL, Knight R, Beiko RG, Huttenhower C. 2013. Predictive functional profiling of microbial communities using 16S rRNA marker gene sequences. Nat Biotechnol 31(9):814-821. DOI: 10.1038/nbt.2676.

Larsen AM, Mohammed HH, Arias CR. 2014. Characterization of the gut microbiota of three commercially valuable warmwater fish species. Journal of applied microbiology 116(6):1396-404 DOI: 10.1111/jam.12475.

Li L, Feng L, Jiang WD, Jiang J, Wu P, Kuang SY, Tang L, Tang WN, Zhang YA, Zhou XQ, Liu Y. 2015. Dietary pantothenic acid deficiency and excess depress the growth, intestinal mucosal immune and physical functions by regulating NF-kappaB, TOR, Nrf2 and MLCK signaling pathways in grass carp 
(Ctenopharyngodon idella). Fish \& shellfish immunology 45(2):399-413 DOI: 10.1016/j.fsi.2015.04.030.

Li W, Liu J, Tan H, Yang C1, Ren L, Liu Q, Wang S, Hu F, Xiao J, Zhao R, Tao M, Zhang C, Qin Q, Liu S. 2018. Genetic effects on the gut microbiota assemblages of hybrid fish from parents with different feeding habits. Front Microbiol 9:2972 DOI: 10.3389/fmicb.2018.02972.

Li Z, Chen F, Huang C, Zheng W, Yu C, Cheng H, Zhou R. 2017. Genome-wide mapping and characterization of microsatellites in the swamp eel genome. Scientific reports 7(1):3157 DOI: 10.1038/s41598-017-033307.

Liem, KF. 1967. Functional morphology of the integumentary, respiratory, and digestive systems of the synbranchoid fish Monopterus albus. Copeia 1967(2): 375-388.

Liu H, Guo X, Gooneratne R, Lai R, Zeng C, Zhan F, Wang W. 2016. The gut microbiome and degradation enzyme activity of wild freshwater fishes influenced by their trophic levels. Scientific reports 6:24340 DOI: $10.1038 /$ srep24340.

Liu J, Bian G, Sun D, Zhu W, Mao S. 2017. Starter Feeding Supplementation Alters Colonic Mucosal Bacterial Communities and Modulates Mucosal Immune Homeostasis in Newborn Lambs. Frontiers in microbiology 8: 429 DOI: 10.3389/fmicb.2017.00429.

Lu HP, Lai YC, Huang SW, Chen HC, Hsieh CH, Yu HT. 2014. Spatial heterogeneity of gut microbiota reveals multiple bacterial communities with distinct characteristics. Scientific reports 4:6185 DOI: 10.1038/srep06185.

Luo Y, Zhang L, Li H, Smidt H, Wright AG, Zhang K, Ding X, Zeng Q, Bai S, Wang J, Li J, Zheng P, Tian G, Cai J, Chen D. 2017. Different Types of Dietary Fibers Trigger Specific Alterations in Composition and Predicted Functions of Colonic Bacterial Communities in BALB/c Mice. Frontiers in microbiology 8:966 DOI: $10.3389 /$ fmicb.2017.00966.

Lv LX, Fang DQ, Shi D, Chen DY, Yan R, Zhu YX, Chen YF, Shao L, Guo FF, Wu WR, Li A, Shi HY, Jiang XW, Jiang HY, Xiao YH, Zheng SS, Li LJ. 2016. Alterations and correlations of the gut microbiome, metabolism and immunity in patients with primary biliary cirrhosis. Environmental microbiology 18(7):2272-86 DOI: 10.1111/1462-2920.13401.

Magoc T, Salzberg SL. 2011. FLASH: fast length adjustment of short reads to improve genome assemblies. Bioinformatics 27: 2957-2963 DOI: 10.1093/bioinformatics/btr507.

Martins P, Cleary DF, Pires AC, Rodrigues AM, Quintino V, Calado R, Gomes NC. 2013. Molecular analysis of bacterial communities and detection of potential pathogens in a recirculating aquaculture system for Scophthalmus maximus and Solea senegalensis. PLoS One 8(11):e80847 DOI: 10.1371/journal.pone.0080847.

Morgan XC, Kabakchiev B, Waldron L, Tyler AD, Tickle TL, Milgrom R, Stempak JM, Gevers D, Xavier RJ, Silverberg, MS, Huttenhower C. 2015. Associations between host gene expression, the mucosal microbiome, and clinical outcome in the pelvic pouch of patients with inflammatory bowel disease. Genome biology 16:67 DOI: 10.1186/s13059-015-0637-x.

Moschen AR, Wieser V, Tilg H. 2012. Dietary Factors: Major Regulators of the Gut's Microbiota. Gut Liver 6(4):411-416 DOI: 10.5009/gnl.2012.6.4.411.

Mountfort DO, Campbell J, Clements KD. 2002. Hindgut fermentation in three species of marine herbivorous fish. Applied and environmental microbiology 68 (3): 1374-1380 DOI: 10.1128/AEM.68.3.1374-1380.2002.

Nadirah M, Ruhil HH, Jalal KC, Najiah M. 2012. Occurrence of Plesiomonas shigelloides in cultured red hybrid 
tilapia (Oreochromis niloticus) from tropical rivers, east coast Malaysia. Pakistan journal of biological sciences 15 (12): 600-603.

Nayak SK. 2010. Probiotics and immunity: a fish perspective. Fish \& shellfish immunology 29(1):2-14 DOI: 10.1016/j.fsi.2010.02.017.

Newaj-Fyzul A, Austin B. 2015. Probiotics, immunostimulants, plant products and oral vaccines, and their role as feed supplements in the control of bacterial fish diseases. Journal of fish diseases 38: 937-955 DOI: $10.1111 /$ jfd.12313.

Nielsen S, Wilkes Walburn J, Verges A, Thomas T, Egan S. 2017. Microbiome patterns across the gastrointestinal tract of the rabbitfish Siganus fuscescens. PeerJ 5: e3317 DOI: 10.7717/peerj.3317.

Patterson AM, Mulder IE, Travis AJ, Lan A, Cerf-Bensussan N, Gaboriau-Routhiau V, Garden K, Logan E, Delday MI, Coutts AGP, Monnais E, Ferraria VC, Inoue R, Grant G, Aminov RI. 2017. Human Gut Symbiont Roseburia hominis Promotes and Regulates Innate Immunity. Frontiers in immunology 8:1166 DOI: $10.3389 /$ fimmu.2017.01166.

Pereira RT, Costa LS, Oliveira IR, Araújo JC, Aerts M, Vigliano FA, Rosa PV. 2015. Tissue Cell 47(2):123-31 DOI: $10.1016 /$ j.tice.2015.01.009.

Petukat S. 1965. Über die arteriellen Gefassstamme bei den Teleosteern. Zool Beitr 11: 437-515.

Piazzon MC, Calduch-Giner JA, Fouz B, Estensoro I, Simó-Mirabet P, Puyalto M, Karalazos V, Palenzuela O, Sitjà-Bobadilla A, Pérez-Sánchez J. 2017. Under control: how a dietary additive can restore the gut microbiome and proteomic profile, and improve disease resilience in a marine teleostean fish fed vegetable diets. Microbiome 5(1):164 DOI: 10.1186/s40168-017-0390-3.

Premalatha N, Gopal NO, Jose PA, Anandham R, Kwon SW. 2015. Optimization of cellulase production by Enhydrobacter sp. ACCA2 and its application in biomass saccharification. Front Microbiol 6:1046 DOI: 10.3389/fmicb.2015.01046.

Rasheeda MK, Rangamaran VR, Srinivasan S, Ramaiah SK, Gunasekaran R, Jaypal S, Gopal D, Ramalingam K. 2017. Comparative profiling of microbial community of three economically important fishes reared in sea cages under tropical offshore environment. Marine genomics 34: 57-65 DOI: 10.1016/j.margen.2017.04.003.

Rudi K, Angell IL, Pope PB, Vik JO, Sandve SR, Snipen LG. 2018. Stable Core Gut Microbiota across the Freshwater-to-Saltwater Transition for Farmed Atlantic Salmon. Appl Environ Microbiol 84(2). pii: e0197417 DOI: 10.1128/AEM.01974-17.

Sakurai T, Sakurai A, Chen Y, Vaisman BL, Amar MJ, Pryor M, Thacker SG, Zhang X, Wang X, Zhang Y, Zhu J, Yang ZH, Freeman LA, Remaley AT. 2017. Dietary alpha-cyclodextrin reduces atherosclerosis and modifies gut flora in apolipoprotein E-deficient mice. Molecular nutrition \& food research 61(8) DOI: 10.1002/mnfr.201600804.

Santos AE, Pedreira MM, Santos TG, Moura GS, Santos JCE, Silva RC. 2016. Development of the digestive system in larvae of the Neotropical fish Prochilodus argenteus (Characiformes, Prochilodontidae). Acta Scientiarum Animal Sciences 38: 9-16.

Sellers RS, Morton D. 2014. The colon: from banal to brilliant. Toxicologic pathology 42(1):67-81 DOI: $10.1177 / 0192623313505930$.

Sharifuzzaman SM, Rahman H, Austin DA, Austin B. 2017. Properties of Probiotics Kocuria SM1 and Rhodococcus SM2 Isolated from Fish Guts. Probiotics and antimicrobial proteins 10(3):534-542 DOI: 10.1007/s12602-017-9290-x. 
Small CM, Milligan-Myhre K, Bassham S, Guillemin K, Cresko WA. 2017. Host Genotype and Microbiota Contribute Asymmetrically to Transcriptional Variation in the Threespine Stickleback Gut. Genome biology and evolution 9: 504-520 DOI: 10.1093/gbe/evx014.

Tap J, Furet JP, Bensaada M, Philippe C, Roth H, Rabot S, Lakhdari O, Lombard V, Henrissat B, Corthier G, Fontaine E, Doré J, Leclerc M. 2015. Gut microbiota richness promotes its stability upon increased dietary fibre intake in healthy adults. Environmental microbiology 17(12):4954-64 DOI: 10.1111/1462-2920.13006.

Wang Q, Garrity GM, Tiedje JM, Cole JR. 2007. Naive Bayesian classifier for rapid assignment of rRNA sequences into the new bacterial taxonomy. Applied and environmental microbiology 73: 5261-5267 DOI: 10.1128/AEM.00062-07.

Wu S, Wang G, Angert ER, Wang W, Li W, Zou H. 2012. Composition, diversity, and origin of the bacterial community in grass carp intestine. PloS one 7, e30440 DOI: 10.1371/journal.pone.0030440.

Wu ZB, Gatesoupe FJ, Li TT, Wang XH, Zhang QQ, Feng DY, Feng YQ, Chen H, Li AH. 2018. Significant improvement of intestinal microbiota of gibel carp (Carassius auratus gibelio) after traditional Chinese medicine feeding. Journal of applied microbiology 124(3):829-841 DOI: 10.1111/jam.13674.

Yan Q, Li J, Yu Y, Wang J, He Z, Van Nostrand JD, Kempher ML, Wu L, Wang Y, Liao L, Li X, Wu S, Ni J, Wang C, Zhou J. 2016. Environmental filtering decreases with fish development for the assembly of gut microbiota. Environmental microbiology 18: 4739-4754 DOI: 10.1111/1462-2920.13365.

Yang S, Duan Y, Zhang J, Zhou J, Liu Y, Du J, Zhao L, Du Z, Han S. 2017. Observational comparisons of intestinal microbiota characterizations, immune enzyme activities, and muscle amino acid compositions of loach in paddy fields and ponds in Sichuan Province. Applied microbiology and biotechnology 101: 47754789 DOI: 10.1007/s00253-017-8167-y.

Ye L, Amberg J, Chapman D, Gaikowski M, Liu WT. 2014. Fish gut microbiota analysis differentiates physiology and behavior of invasive Asian carp and indigenous American fish. ISME $J$ 8(3):541-51 DOI: 10.1038/ismej.2013.181.

Yin, J., Han, H., Li, Y., Liu, Z., Zhao, Y., Fang, R., Huang, X., Zheng, J., Ren, W., Wu, F., Liu, G., Wu, X., Wang, K., Sun, L., Li, C., Li, T., Yin, Y., 2017. Lysine Restriction Affects Feed Intake and Amino Acid Metabolism via Gut Microbiome in Piglets. Cellular physiology and biochemistry 44(5): 1749-1761 DOI: $10.1159 / 000485782$.

Zothanpuia, Passari AK, Gupta VK, Singh BP, 2016. Detection of antibiotic-resistant bacteria endowed with antimicrobial activity from a freshwater lake and their phylogenetic affiliation. PeerJ 4:e2103 DOI: 10.7717/peerj.2103.

Peer) reviewing PDF | (2018:02:24034:1:2:NEW 9 Oct 2019) 


\section{Figure 1}

The observed OTU numbers, unique and sharedOTUs in different gut compartments $(n=4)$.

(A) Bar plot shows the observed OTU numbers in midgut content (MC), midgut mucosa (MM), hindgut content (HC), hindgut mucosa (HM), and stools (S). (B) Venn diagram displays the number of shared and unique OTUs among midgut content (MC), hindgut content (HC), and stools (S). (C) Venn diagram displays the number of shared and unique OTUs between midgut mucosa (MM) and hindgut mucosa (HM). (D) Venn diagram displays the number of core OTUs shared by all gut compartments. 


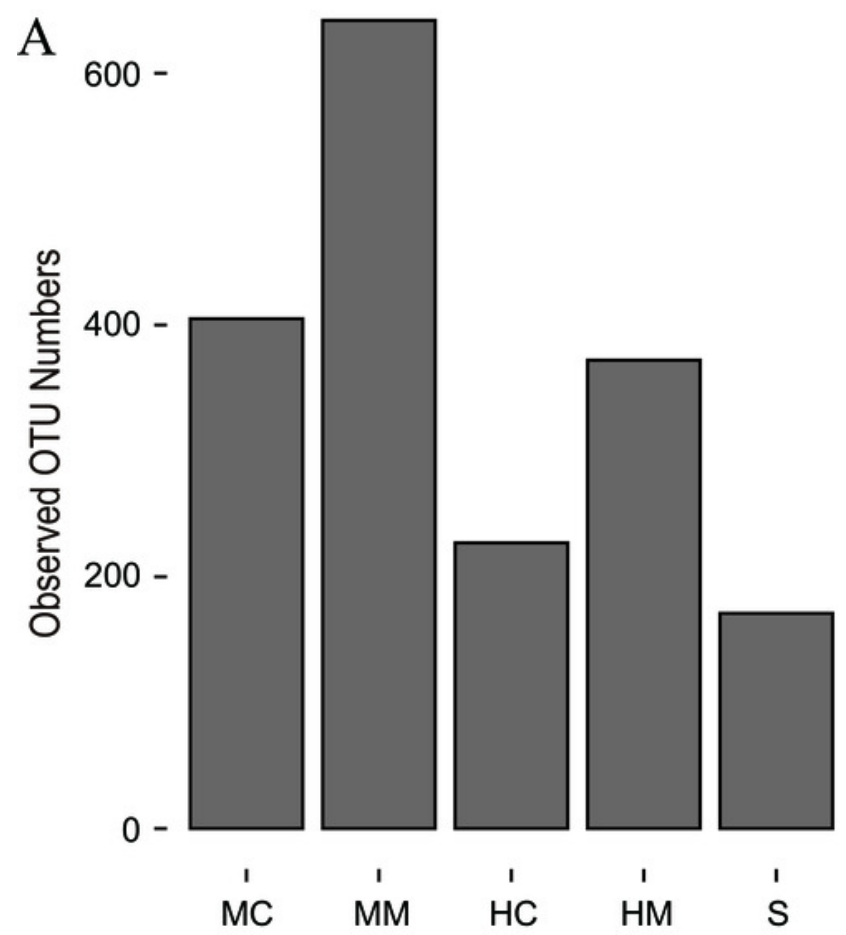

B

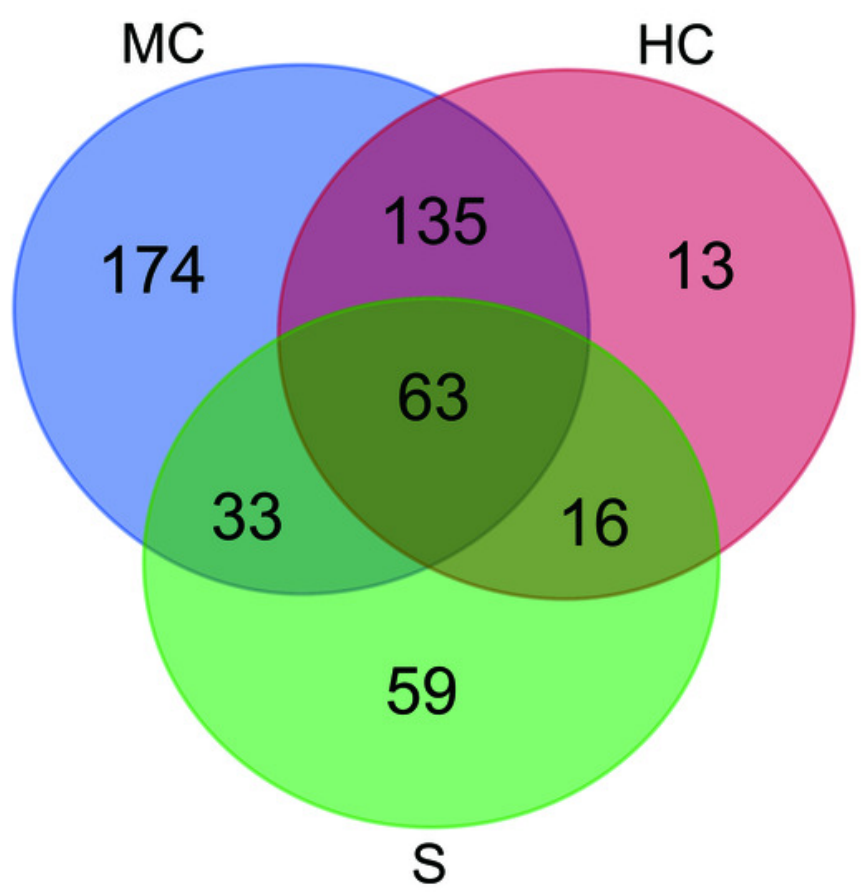

C

D

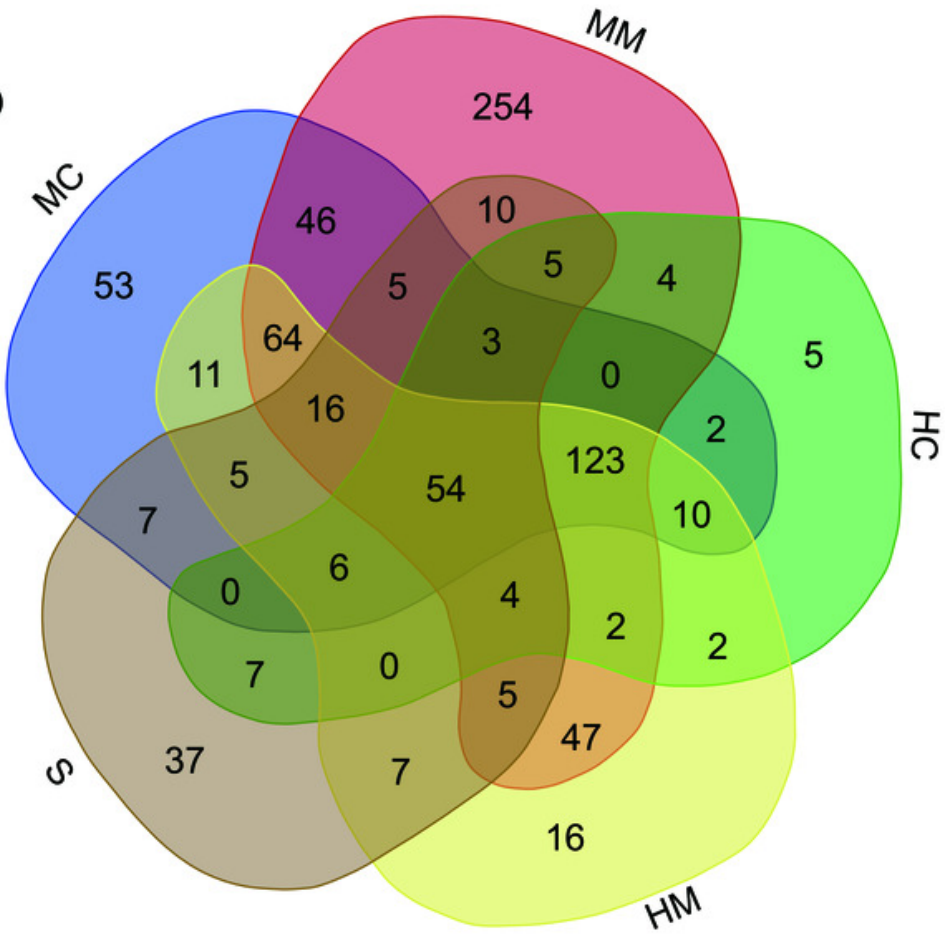


Figure 2

Principal Coordinate Analysis (PCOA) ofmicrobial community in different gut compartments based on the UnweightedUniFrac distance matrix $(n=4)$.

The individual samples are color- and shape-coordinated according to the gut compartment. MC : midgut content, MM: midgut compartment, $\mathrm{HC}$ : hindgut content , $\mathrm{HM}$ : hindgut mucosa, S: stools

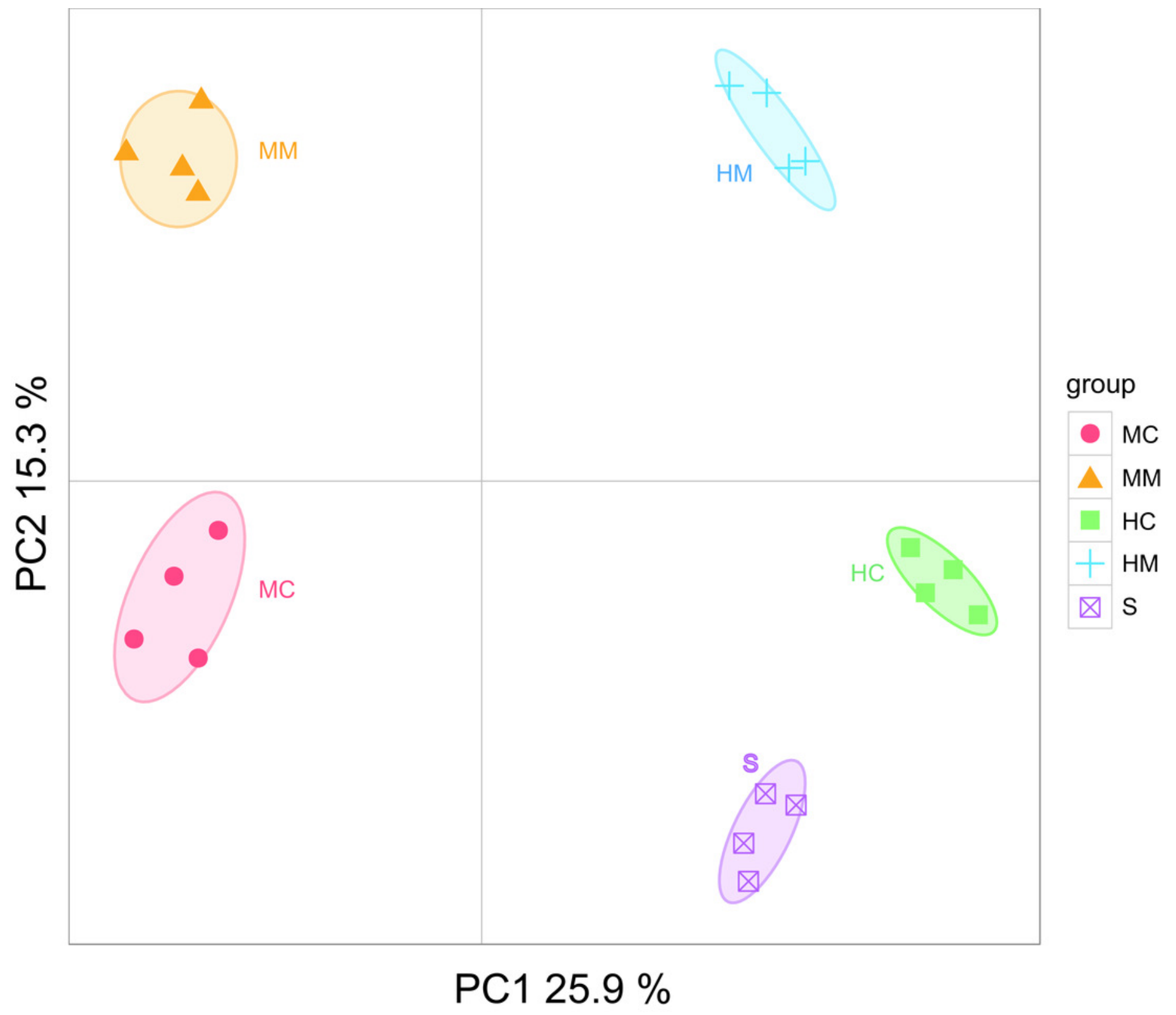




\section{Figure 3}

Gut microbial compositions at the phylumlevel and genus level $(n=4)$.

(A) Each bar represents average relative abundance of each phylum of gut microbiota in midgut content $(M C)$, midgut mucosa $(M M)$, hindgut content $(H C)$, hindgut mucosa $(H M)$, and stools (S). (B) Each bar represents average relative abundance of each genra of gut microbiota in midgut content (MC), midgut mucosa (MM), hindgut content $(\mathrm{HC})$, hindgut mucosa (HM), and stools (S).
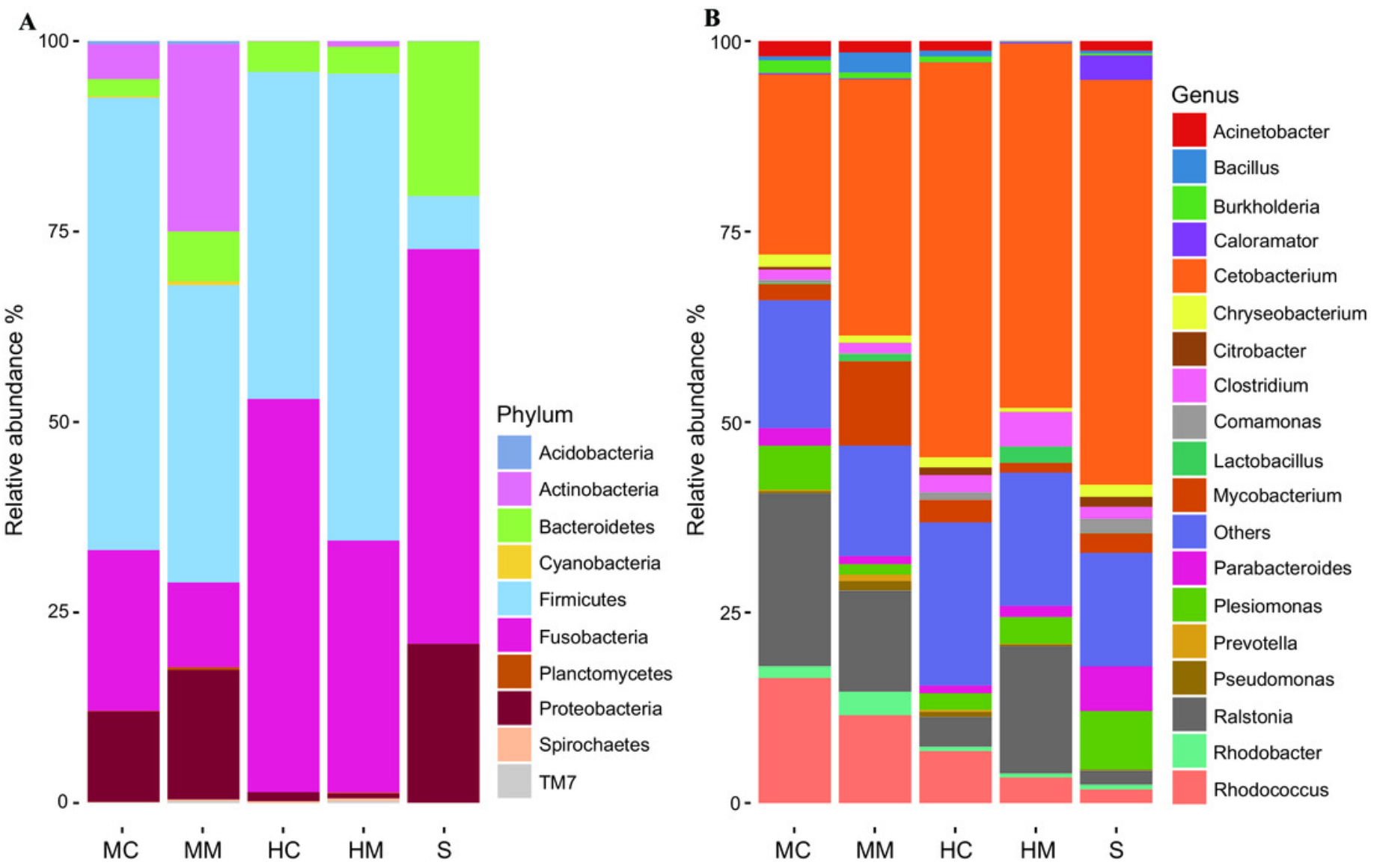
Figure 4

Gut compartment-specific enriched core OTUs $(n=4)$.

Heat map shows core OTUs significantly varied in abundances in different gut compartments (cell note on the heat map represents differentially abundant OTUs annotated to microbial taxa).
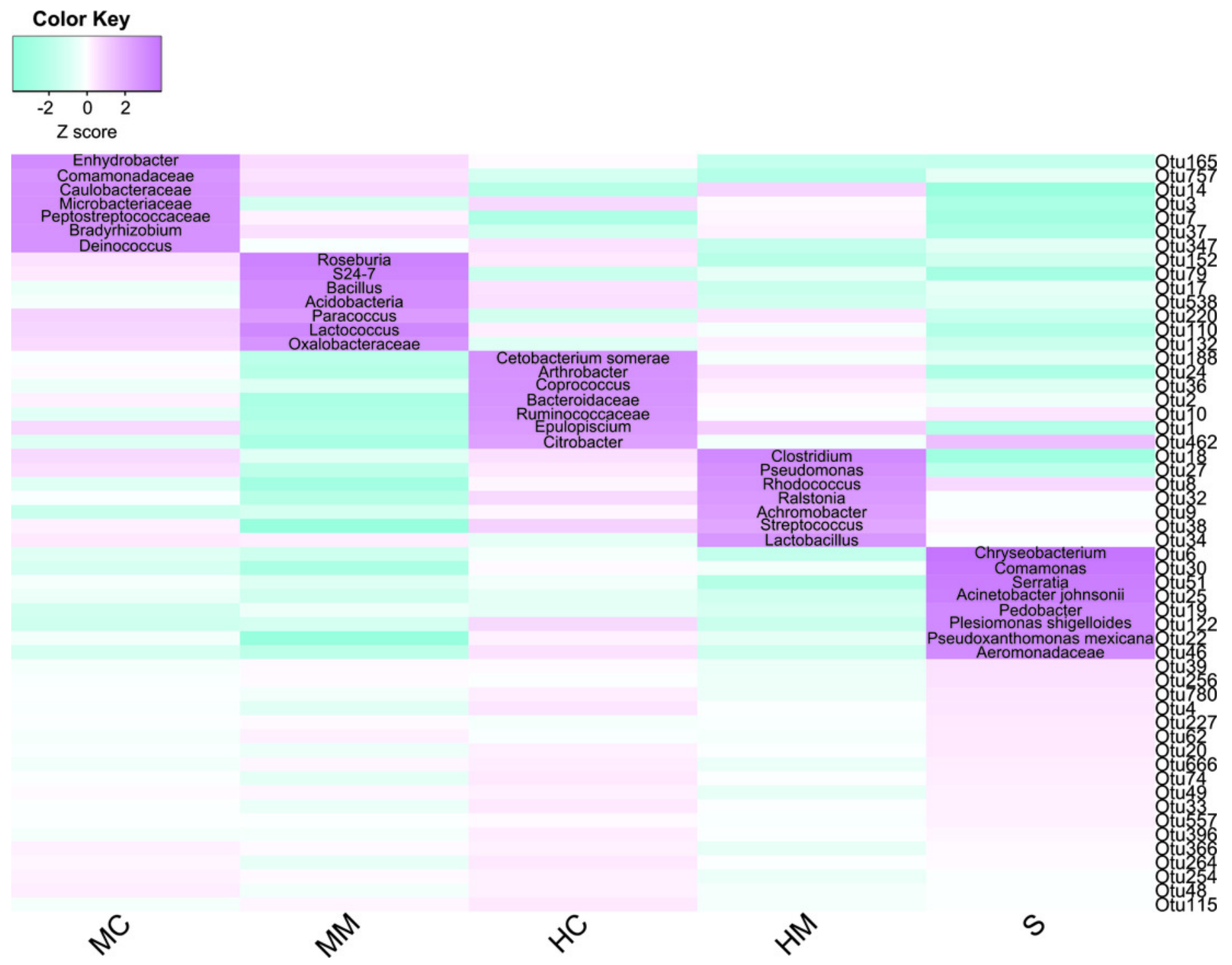


\section{Figure 5}

Comparison in the abundance of gutmicrobial potential function capacities in different gut compartments $(n=4)$.

Heat map shows the abundances of gut microbial KEGG pathways (level 3) significantly changed in different gut compartments (cell note on the heat map represents differentially abundant KEGG pathways at level 2 ).

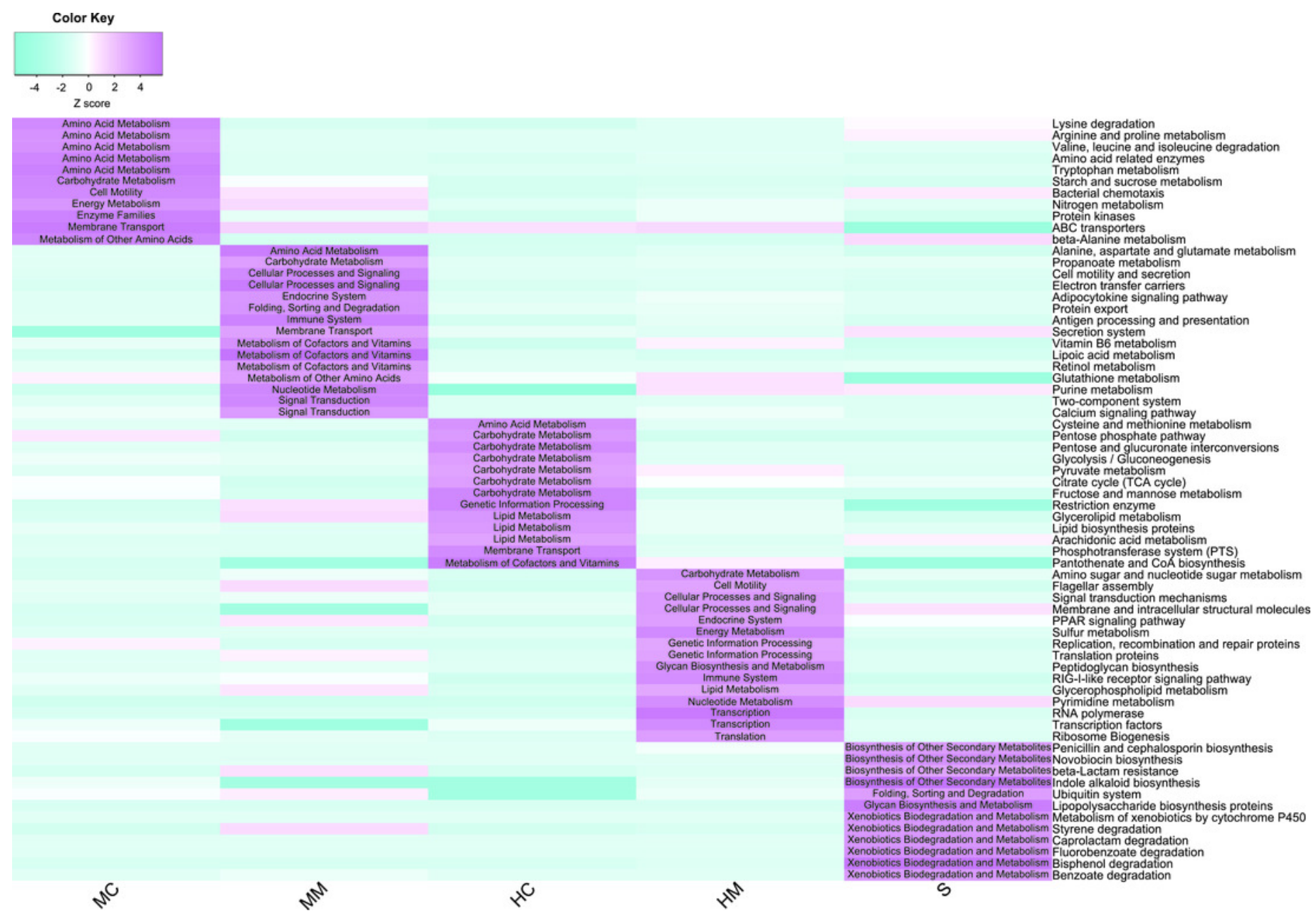

